\title{
Recognition and management of anxiety syndromes
}

\author{
HANS-ULRICH WITTCHEN
}

The past two decades have witnessed an increasing interest in research on anxiety syndromes and disorders. Significant advances in basic and clinical research, accompanied by animated ongoing discussions about the most appropriate, reliable and valid way to classify and assess anxiety disorders, have changed our understanding of anxiety disorders and affected (or some might say confused) our research and treatment practice.

Disregarding the pros and cons of this development (Andrews \& Wittchen, 1995), the increasing recognition that a more sophisticated subtyping of anxiety disorders, as now codified by the 10 th revision of the International Classification of Diseases (ICD-10; World Health Organization, 1991) or the 4th revision of the Diagnostic and Statistical Manual of Mental Disorders (DSM-IV; American Psychiatric Association, 1994), has at least stimulated a lot of research. Numerous epidemiological studies have investigated the size of the problem in the general population and in primary care settings, and some studies have demonstrated the tremendous economic burden on society due to anxiety disorders. Hundreds of clinical studies have demonstrated the effectiveness and tried to explore further the mechanisms of actions of new disorderspecific pharmacological and psychological treatments, and there has been considerable research activity aiming at an improvement of diagnostic accuracy in measuring symptoms, disabilities, comorbid complications and risk factors.

This supplement brings together nine contributions addressing each of these areas, reviewing and discussing most recent data, new research evidence, and critical issues requiring further investigation. To set the stage as well as to preview some of the major implications of the following papers, three unresolved issues and continuing challenges are highlighted in this editorial: (a) how real is the gap between high prevalence rates of anxiety disorders in the community and very low treatment rates, (b) how poor are recognition rates in primary care, and (c) does treatment research offer new solutions?

\section{THE SIZE OF THE PROBLEM: EVIDENCE FOR A CONSIDERABLE DEGREE OF UNMET NEED}

In spite of the existence of reliable diagnostic instruments and the availability of acceptable and effective treatments for anxiety disorders, general population studies in several countries (Canada, Katz et al, 1994; Offord et al, 1994; Great Britain, Meltzer et al, 1994; US, Kessler et al, 1994, 1997; Germany, Wittchen et al, 1998) have convergently shown that almost two-thirds of those affected by anxiety disorders receive no professional treatment, and even fewer receive qualified specialist mental health treatment. Does this mean that the vast majority of the $10-15 \%$ of the population with anxiety disorders are in need of treatment without receiving appropriate professional help and attention? This conclusion would only be justified if we could simply equate the diagnosis of an anxiety disorder directly with specific professional treatment needs. This seems to be a questionable assumption in the case of anxiety disorders (Regier et al, 1998). Unlike other mental disorders, such as schizophrenia, where such an assumption might receive greater professional consensus (Häfner, 1979), at least some forms of anxiety disorders might have significant spontaneous remission rates or might have fluctuating courses in terms of severity of anxiety symptoms, related impairments, and risk for complications. Thus, a yet unknown proportion of anxiety cases seems to improve over time without interventions or by low impact interventions such as selfhelp manuals or psychoeducation. The substantial proportion of what is labelled 'placebo' response in randomised trials, where the inactive ingredient consists only of information and comprehensive selfmonitoring tools, seems to support this assumption (Bond \& Lader, 1996).

In determining the degree of unmet need in anxiety disorders in the population, several additional considerations might be necessary beyond the mere establishment of a reliable diagnosis on the one hand and the provision of services on the other. Unfortunately the available epidemiological studies have failed so far to provide sufficiently detailed data (Regier et al, 1998). This is probably due to the fact that generally accepted standards and instruments for need evaluation as well as disease-specific disabilities are largely unavailable. To conclude, our current knowledge about unmet needs for anxiety treatments in the population is largely deficient, and this unsatisfactory situation will probably remain unchanged in the near future. Until such approaches eventually become available, firm knowledge about the natural course, risks and developmental complications (i.e. comorbidity) of anxiety disorders are of key importance. This type of indirect evidence might at least offer some general guidance.

Four of the contributions in this supplement provide such data, all underlining the clinical significance and considerable adverse developmental implications of anxiety disorders. In addition to the already mentioned impressive and sophisticated documentation of the economic burden of anxiety disorders by Rice and colleagues, Regier and colleagues report cross-sectional and longitudinal data from the Epidemiological Catchment Area study. Within this complex prospective design it is shown that temporally primary anxiety disorders, especially simple and social phobias, appear to have an early onset with potentially severe consequences, predisposing to a significant vulnerability to major depression and addictive disorders. Schatzberg and colleagues confirm these epidemiological findings of early primary anxiety disorders as risk/ vulnerability factors for depression in a clinical convenience sample, elaborating risk constellations and speculating about various 'pathways' and explanatory constructs of the observed comorbidity patterns. Emmanuel and collaborators supplement these study findings concerning comorbidity by a systematic review of eight carefully chosen treatment studies. In 
agreement with the previous studies a poorer outcome of comorbid anxiousdepressive patients compared with separate anxiety and depressive disorders is demonstrated. Noteworthy is also a somewhat better outcome in patients with depressive disorders compared with anxiety disorders.

Although these contributions do not directly determine the degree of unmet need for anxiety disorders, they at least demonstrate convincingly once again that anxiety disorders are serious mental disorders. Even simple and social phobias, frequently regarded as not being clinically significant disorders at all, carry substantial risks for development of severe depressive disorders and seem to have an unfavourable course and outcome. One key conclusion and challenge discussed, namely to offer effective early intervention and preventive strategies to reduce such risks and morbidity, presents a significant challenge to epidemiology, clinical psychiatry and public health research, and it is unlikely that an appropriate answer will be found in the near future.

\section{POOR RECOGNITION AND TREATMENT RATES IN PRIMARY CARE}

Several general population surveys have shown that at least every second case with full-blown (threshold) anxiety disorders has told, at least once, their primary care doctor about their condition. Yet the actual treatment rates are extremely low (Wittchen, 1998). The paper by Weiller and colleagues is another confirmation of this information based on data from five European centres, involved in the prospective multicentre World Health Organization landmark study on 'Psychological problems in general health care' (Üstün \& Sartorius, 1995). Based on a large representative sample of 1973 primary care attenders the authors find that about $10 \%$ of all primary care patients present with significant current anxiety syndromes (predominantly generalised anxiety) of considerable severity, with significant social disability and elevated disability days in the past month. They further confirm earlier findings, that only about one-third was correctly recognised as having an anxiety disorder and even fewer $(60.5 \%$ of the recognised anxiety cases) received any form of intervention (mostly counselling). What makes this report important is: (a) the (fairly disappointing) demonstration that very rarely are effective, scientifically proven treatments provided; fewer than $10 \%$ of all anxiety disorders in primary care offices receive recommended anxiety treatment; (b) the finding, that even though most patients come to their doctor specifically because they are suffering from anxiety symptoms, and in spite of revealing significant social disabilities, they are not recognised.

This underlines the case that even if the anxiety disorder is the primary reason for contact, doctors in general health care fail to recognise them. This failure is particularly pronounced in the most frequent condition, namely in generalised anxiety disorder (GAD). Given the key role primary care doctors play in most health care systems this stresses the need for a continued search for more successful strategies to improve this situation. Over the past decade the many more or less structured attempts to reach the goal of improved recognition have obviously not been tremendously successful.

As one potentially helpful addition to this challenging undertaking of improving recognition skills, the contribution by Wittchen \& Boyer suggests the use of prototypical screening questionnaires. This type of questionnaire, unlike general caseness instruments (indicating the likelihood of being a psychiatric case) such as the General Health Questionnaire, is tailored strictly along the explicit diagnostic criteria for specific anxiety disorders. Referring to the poor recognition of generalised anxiety by primary care providers the authors developed and pilot-tested a short and efficient 15 -item screening questionnaire, shown to be highly sensitive and specific for threshold and subthreshold generalised anxiety disorders and sensitive (while less specific) for other disorders. Although this approach is clearly in need of a more comprehensive psychometric and clinical evaluation it might prove to be an easy to use, acceptable and more specific tool for improving recognition skills of primary care providers.

\section{STRATEGIES TO IMPROVE UNMET NEEDS IN ANXIETY DISORDERS: PRIORITIES IN TREATMENT DEVELOPMENT}

Because of the large proportion of the population affected by at least one anxiety disorder, even among children and adolescents (Wittchen et al, 1998), it is unlikely that highly sophisticated psychological and drug treatment regimens (although much needed for the most severe) will significantly affect morbidity and disability rates in the population. This type of highly sophisticated research, however, might provide urgently needed insight into mechanisms of actions and the identification of the most active ingredients, also potentially essential for designing low-impact interventions in the future. Aufdembrinke in his exploration of beta-carbolines as a potential new class of drugs in the treatment of generalised anxiety, Rickels \& Schweizer with their concepts of short intermittent drug treatment, and in particular the paper by Lader \& Bond, are three examples of present search strategies in anxiety disorder. The latter give a stimulating summary of state of the art issues and critical concerns in research of psychological and drug therapies, and provide findings from combined drug and psychological intervention in GAD patients. There seem to be some indications for additional benefits of combined treatments (speed of onset and lasting remissions); however, they also demonstrate the difficulty in showing superiority of combined treatments $v$. two already effective treatments.

Given the above-mentioned unmet needs, poor recognition by the de facto health care system and high proportion of inadequate treatments of those recognised, these and all contributions to this supplement leave open the most challenging questions: how can those in need be reached? How can effective treatments be translated more appropriately into routine care? And what active ingredients of effective anti-anxiety treatments are candidates for those preventive and early interventions suggested? The search for answers to these challenging questions will require close collaboration across the disciplinary borders of clinical psychiatry, psychology, epidemiology and public health research.

\section{REFERENCES}

American Psychiatric Association (1994) Diagnostic and Statistical Manual of Mental Disorders (4th edn) (DSM-IV). Washington, DC: APA.

Andrews, G. \& Wittchen, H.-U. (1995) Clinical practice, measurement and information technology. Psychological Medicine, 25, 443-446.

Bond, A. J. \& Lader, M. H. (1996) Understanding Drug Treatment in Mental Health Care. Chichester: Wiley. 
Häfner, H. (ed.) (1979) Estimating Needs for Mental Health Care. A Contribution of Epidemiology. Berlin: Springer.

Katz, S. J., Kessler, R. C., Frank, R. G., et al (1997) The use of outpatient mental health services in the United States and Ontario: The impact of mental morbidity and perceived need of care. American Journal of Public Health, 87, I136-1143.

Kessler, R. C., McGonagle, K. A., Zhao, S., et al (1994) Lifetime and 12-month prevalence of DSM-III-R psychiatric disorders in the United States: Results from the National Comorbidity Survey. Archives of General Psychiatry, 51, 8-19.

_, Frank, R. G., Edlund, M., et al (1997) Differences in the use of psychiatric outpatient services between the U.S. and Ontario. New England journal of Medicine, 336, $55 \mid-557$.

HANS-ULRICH WITTCHEN, PhD, Max Planck Institute of Psychiatry, 8004 München, Kraepelinstr. 2, Germany

Offord, D., Boyle, M., Campbell, D., et al (1994) Ontario Health Survey 1990. Mental Health Supplement. Toronto: Ministry of Health.

Regier, D. A., Kaelber, C.T., Rae, D. S., et al (1998) Limitations of diagnostics criteria and assessment instruments for mental disorders. Archives of General Psychiatry, 55, 109-115.

Üstün, T. B. Sartorius, N. (1995) Mental Health in General Health Care. Chichester: Wiley.

Wittchen, H.-U. (1998) Met and unmet need for interventions in community cases with anxiety disorders. In Met and Unmet Needs (eds G. Andrews \& S. Henderson). Cambridge University Press, in press.
Nelson, G. B. \& Lachner, G. (1998) Prevalence of mental disorders and psychosocial impairments in adolescents and young adults. Psychological Medicine, $\mathbf{2 8}$ $109-126$.

World Health Organization (1990) Composite International Diagnostic Interview (CIDI). Geneva: World Health Organization, Division of Mental Health.

- (1992) The Tenth Revision of the International Classification of Diseases and Related Health Problems (ICD-10). Geneva: WHO 